Pareto, Anonymity, and Independence: Four Alternatives

Donald E. Campbell

College of William and Mary

Jerry S. Kelly

Syracuse University

College of William and Mary

Department of Economics

Working Paper Number 39

August 2006

Forthcoming in Social Choice and Welfare. 
COLLEGE OF WILLIAM AND MARY

DEPARTMENT OF ECONOMICS

WORKING PAPER \# 39

August 2006

\title{
Pareto, Anonymity, and Independence: Four Alternatives
}

\begin{abstract}
For four alternatives and an even number of individuals, we prove a conjecture in a companion paper: It is impossible for a social choice rule to satisfy all of (1) Pareto, (2) anonymity, (3) full domain, and (4) independence of some alternative, a relaxation of Arrow's IIA.
\end{abstract}

JEL Codes: D70, D71

Keywords: Pareto, anonymity, independence

Donald E. Campbell

Department of Economics

College of William and Mary

Williamsburg, VA 23187-8795

decamp@wm.edu
Jerry S. Kelly

Department of Economics

Syracuse University

Syracuse, NY 13245-1090

jskelly@maxwell.syr.edu 


\title{
Pareto, Anonymity, and Independence: Four Alternatives ${ }^{1}$
}

\author{
Donald E. Campbell and Jerry S. Kelly
}

\section{Introduction.}

A social welfare function $\mathrm{f}$ defined for the set $\mathrm{X}$ of alternatives satisfies Independence of Some Alternative (ISA) if for every distinct pair $\{\mathrm{x}, \mathrm{y}\}$ of alternatives there is some $\mathrm{z} \in \mathrm{X}$ such that $\mathrm{f}(\mathrm{p})|\{\mathrm{x}, \mathrm{y}\}=\mathrm{f}(\mathrm{q})|\{\mathrm{x}, \mathrm{y}\}$ if the profiles $\mathrm{p}$ and $\mathrm{q}$ agree on $X \backslash\{z\}$. (When $X=\{x, y, z\}$ ISA is equivalent to IIA.) This paper proves, when $X$ has exactly four alternatives, a conjecture from Campbell and Kelly (2005) where definitions and notation are provided.

CONJECTURE: For any $\mathrm{X}$, there does not exist a rule satisfying all of: Independence of Some Alternative, Pareto, full domain, transitivity, and anonymity.

The relevance map $\psi$ for social welfare function f identifies the smallest subset $\psi(\{x, y\})$ of $X$ such that $f(p)|\{x, y\}=f(q)|\{x, y\}$ whenever $p$ and $q$ agree on $\psi(\{x, y\})$. Campbell and Kelly (2000) show that if $X$ is finite there will be a smallest such set. Then Campbell and Kelly (2005) proved the above conjecture for the case of relevance maps that satisfy exclusion: A triple $\{\mathrm{x}, \mathrm{y}, \mathrm{z}\}$ satisfies exclusion if $\mathrm{x} \notin \psi(\mathrm{y}, \mathrm{z}), \mathrm{y} \notin \psi(\mathrm{x}, \mathrm{z})$, and $\mathrm{z} \notin \psi(\mathrm{x}, \mathrm{y})$ and relevance map $\psi$ satisfies exclusion if there is at least one triple satisfying exclusion. If $\mathrm{x} \notin \Psi(\{\mathrm{y}, \mathrm{z}\}), \mathrm{y} \notin \Psi(\{\mathrm{x}, \mathrm{z}\})$, and $\mathrm{z} \notin \Psi(\{\mathrm{x}, \mathrm{y}\})$ then we can restrict attention to the profiles for which the top three alternatives in each individual ordering are $\mathrm{x}, \mathrm{y}$, and $z$, with the remaining alternatives in fixed positions relative to each other and to $x, y$, and $z$. Then over this sub-family of profiles, IIA is satisfied as we move $\mathrm{x}, \mathrm{y}$, and $\mathrm{z}$ around, and that leads to dictatorship of the restriction of $\mathrm{f}$ to the subfamily if $\mathrm{f}$ satisfies Pareto. This implies that $\mathrm{f}$ violates anonymity.

But there are relevance maps that fail exclusion but still satisfy ISA. Here we prove the more general result for the case with an even number of individuals and four alternatives: $X=\{w, x, y, z\}$. In the first three sections, we treat the case of just two individuals. This is generalized in Section 4.

We start by determining the relevance maps we need to consider. For a rule $f$ that satisfies ISA, Pareto, and full domain, Campbell and Kelly (2005) have shown that if the relevance map for $\mathrm{f}$ is simple, i.e., if $\{x, y\} \subseteq \psi(u, v)$ implies $\psi(\mathrm{x}, \mathrm{y}) \subseteq \psi(\mathrm{u}, \mathrm{v})$, then it satisfies exclusion. So all relevance maps that violate exclusion may be found among those that fail simplicity. Without loss of generality, we may consider as a generic case of simplicity violation with ISA holding:

$$
\begin{aligned}
& \psi(\{x, y\})=\{x, y, z\} \\
& \psi(\{y, z\})=\{y, z, w\}
\end{aligned}
$$

To exploit this, we draw on a result from Campbell and Kelly (2000):

1 We would like to thank two referees who found a significant error in an earlier version. 
Map Theorem. For any transitive-valued Paretian social welfare function $\mathrm{f}$ on a full domain, where $\mathrm{x}, \mathrm{y}$, and $\mathrm{w}$ are any three distinct alternatives in $X$, it cannot be the case that both $\psi(x, y)=\{x, y\}$ and $\psi(y, w)=\{y, w\} \cup B$ where $\mathrm{x} \notin \mathrm{B} \neq \varnothing$.

Returning to the relevance map satisfying $(\star)$, if $\psi(\{y, w\})=\{y, w\}$, that, together with $\psi(\{x, y\})=\{x, y, z\}$, would violate the map theorem, so $\psi(\{y, w\})=\{y, w, x\}$ or $\psi(\{y, w\})=\{y, w, z\}$. Similarly, $\psi(\{x, z\})=\{x, z\}$ plus $\psi(\{y, z\})$ $=\{\mathrm{y}, \mathrm{z}, \mathrm{w}\}$ violate the map theorem so $\psi(\{\mathrm{x}, \mathrm{z}\})=\{\mathrm{x}, \mathrm{z}, \mathrm{y}\}$ or $\psi(\{\mathrm{x}, \mathrm{z}\})=\{\mathrm{x}, \mathrm{z}, \mathrm{w}\} . \quad \psi(\{\mathrm{x}, \mathrm{w}\})=\{\mathrm{x}, \mathrm{w}\}$ plus $\psi(\{\mathrm{x}, \mathrm{y}\})=$ $\{\mathrm{x}, \mathrm{y}, \mathrm{z}\}$ also violate the map theorem, so $\psi(\{\mathrm{x}, \mathrm{w}\})=\{\mathrm{x}, \mathrm{w}, \mathrm{y}\}$ or $\psi(\{\mathrm{x}, \mathrm{w}\})=\{\mathrm{x}, \mathrm{w}, \mathrm{z}\} . \psi(\{\mathrm{z}, \mathrm{w}\})=\{\mathrm{z}, \mathrm{w}\}$ is consistent with both $\psi(\{\mathrm{x}, \mathrm{y}\})=\{\mathrm{x}, \mathrm{y}, \mathrm{z}\}$ and $\psi(\{\mathrm{y}, \mathrm{z}\})=\{\mathrm{y}, \mathrm{z}, \mathrm{w}\}$, but would be inconsistent with $\psi(\{\mathrm{y}, \mathrm{w}\})=\{\mathrm{y}, \mathrm{w}, \mathrm{x}\}$ or $\psi(\{\mathrm{x}, \mathrm{w}\})=$ $\{\mathrm{x}, \mathrm{w}, \mathrm{y}\}$ or $\psi(\{\mathrm{x}, \mathrm{z}\})=\{\mathrm{x}, \mathrm{z}, \mathrm{y}\}$. So the possible cases are either:

\begin{tabular}{|c|c|}
\hline $\mathrm{S}$ & $\Psi(\mathrm{S})$ \\
\hline$\{\mathrm{x}, \mathrm{y}\}$ & $\{\mathrm{x}, \mathrm{y}, \mathrm{z}\}$ \\
$\{\mathrm{y}, \mathrm{z}\}$ & $\{\mathrm{y}, \mathrm{z}, \mathrm{w}\}$ \\
$\{\mathrm{x}, \mathrm{w}\}$ & $\{\mathrm{x}, \mathrm{w}, \mathrm{z}\}$ \\
$\{\mathrm{y}, \mathrm{w}\}$ & $\{\mathrm{y}, \mathrm{w}, \mathrm{z}\}$ \\
$\{\mathrm{z}, \mathrm{w}\}$ & $\{\mathrm{z}, \mathrm{w}\}$ \\
$\{\mathrm{x}, \mathrm{z}\}$ & $\{\mathrm{x}, \mathrm{z}, \mathrm{w}\}$ \\
& \\
\hline
\end{tabular}

or one of the 16 maps consistent with

\begin{tabular}{|c|c|}
\hline $\mathrm{S}$ & $\Psi(\mathrm{S})$ \\
$\{\mathrm{x}, \mathrm{y}\}$ & $\{\mathrm{x}, \mathrm{y}, \mathrm{z}\}$ \\
$\{\mathrm{y}, \mathrm{z}\}$ & $\{\mathrm{y}, \mathrm{z}, \mathrm{w}\}$ \\
$\{\mathrm{x}, \mathrm{w}\}$ & $\{\mathrm{x}, \mathrm{w}, \mathrm{y}\}$ or $\{\mathrm{x}, \mathrm{w}, \mathrm{z}\}$ \\
$\{\mathrm{y}, \mathrm{w}\}$ & $\{\mathrm{y}, \mathrm{w}, \mathrm{x}\}$ or $\{\mathrm{y}, \mathrm{w}, \mathrm{z}\}$ \\
$\{\mathrm{z}, \mathrm{w}\}$ & $\{\mathrm{z}, \mathrm{w}, \mathrm{x}\}$ or $\{\mathrm{z}, \mathrm{w}, \mathrm{y}\}$ \\
$\{\mathrm{x}, \mathrm{z}\}$ & $\{\mathrm{x}, \mathrm{y}, \mathrm{z}\}$ or $\{\mathrm{x}, \mathrm{z}, \mathrm{w}\}$ \\
& \\
\hline
\end{tabular}

Note that many of these cases can be ruled out as satisfying exclusion. Altogether, seven of 17 cases are eliminated in this way. 
The ten remaining maps are as follows:

1.

\begin{tabular}{|c|c|}
\hline $\mathrm{S}$ & $\Psi(\mathrm{S})$ \\
\hline & \\
$\{\mathrm{x}, \mathrm{y}\}$ & $\{\mathrm{x}, \mathrm{y}, \mathrm{z}\}$ \\
$\{\mathrm{y}, \mathrm{z}\}$ & $\{\mathrm{y}, \mathrm{z}, \mathrm{w}\}$ \\
$\{\mathrm{x}, \mathrm{w}\}$ & $\{\mathrm{x}, \mathrm{w}, \mathrm{y}\}$ \\
$\{\mathrm{y}, \mathrm{w}\}$ & $\{\mathrm{y}, \mathrm{w}, \mathrm{x}\}$ \\
$\{\mathrm{z}, \mathrm{w}\}$ & $\{\mathrm{z}, \mathrm{w}, \mathrm{x}\}$ \\
$\{\mathrm{x}, \mathrm{z}\}$ & $\{\mathrm{x}, \mathrm{y}, \mathrm{z}\}$ \\
& \\
\hline
\end{tabular}

2.

\begin{tabular}{|c|c|}
\hline$S$ & $\psi(S)$ \\
\hline$\{\mathrm{x}, \mathrm{y}\}$ & $\{\mathrm{x}, \mathrm{y}, \mathrm{z}\}$ \\
\hline$\{y, z\}$ & $\{\mathrm{y}, \mathrm{z}, \mathrm{w}\}$ \\
\hline$\{x, w\}$ & $\{\mathrm{x}, \mathrm{w}, \mathrm{y}\}$ \\
\hline$\{\mathrm{y}, \mathrm{w}\}$ & $\{\mathrm{y}, \mathrm{w}, \mathrm{x}\}$ \\
\hline$\{\mathrm{z}, \mathrm{w}\}$ & $\{\mathrm{z}, \mathrm{w}, \mathrm{x}\}$ \\
\hline$\{\mathrm{x}, \mathrm{z}\}$ & $\{\mathrm{x}, \mathrm{Z}, \mathrm{w}\}$ \\
\hline
\end{tabular}

3.

\begin{tabular}{|c|c|}
\hline $\mathrm{S}$ & $\Psi(\mathrm{S})$ \\
\hline$\{\mathrm{x}, \mathrm{y}\}$ & $\{\mathrm{x}, \mathrm{y}, \mathrm{z}\}$ \\
$\{\mathrm{y}, \mathrm{z}\}$ & $\{\mathrm{y}, \mathrm{z}, \mathrm{w}\}$ \\
$\{\mathrm{x}, \mathrm{w}\}$ & $\{\mathrm{x}, \mathrm{w}, \mathrm{y}\}$ \\
$\{\mathrm{y}, \mathrm{w}\}$ & $\{\mathrm{y}, \mathrm{w}, \mathrm{x}\}$ \\
$\{\mathrm{z}, \mathrm{w}\}$ & $\{\mathrm{z}, \mathrm{w}, \mathrm{y}\}$ \\
$\{\mathrm{x}, \mathrm{z}\}$ & $\{\mathrm{x}, \mathrm{z}, \mathrm{w}\}$ \\
\hline
\end{tabular}

4.

\begin{tabular}{|c|c|}
\hline $\mathrm{S}$ & $\Psi(\mathrm{S})$ \\
\hline & \\
$\{\mathrm{x}, \mathrm{y}\}$ & $\{\mathrm{x}, \mathrm{y}, \mathrm{z}\}$ \\
$\{\mathrm{y}, \mathrm{z}\}$ & $\{\mathrm{y}, \mathrm{z}, \mathrm{w}\}$ \\
$\{\mathrm{x}, \mathrm{w}\}$ & $\{\mathrm{x}, \mathrm{w}, \mathrm{y}\}$ \\
$\{\mathrm{y}, \mathrm{w}\}$ & $\{\mathrm{y}, \mathrm{w}, \mathrm{z}\}$ \\
$\{\mathrm{z}, \mathrm{w}\}$ & $\{\mathrm{z}, \mathrm{w}, \mathrm{x}\}$ \\
$\{\mathrm{x}, \mathrm{z}\}$ & $\{\mathrm{x}, \mathrm{y}, \mathrm{z}\}$ \\
& \\
\hline
\end{tabular}


5.

\begin{tabular}{|c|c|}
\hline $\mathrm{S}$ & $\psi(\mathrm{S})$ \\
\hline$\{\mathrm{x}, \mathrm{y}\}$ & $\{\mathrm{x}, \mathrm{y}, \mathrm{z}\}$ \\
$\{\mathrm{y}, \mathrm{z}\}$ & $\{\mathrm{y}, \mathrm{z}, \mathrm{w}\}$ \\
$\{\mathrm{x}, \mathrm{w}\}$ & $\{\mathrm{x}, \mathrm{w}, \mathrm{y}\}$ \\
$\{\mathrm{y}, \mathrm{w}\}$ & $\{\mathrm{y}, \mathrm{w}, \mathrm{z}\}$ \\
$\{\mathrm{z}, \mathrm{w}\}$ & $\{\mathrm{z}, \mathrm{w}, \mathrm{x}\}$ \\
$\{\mathrm{x}, \mathrm{z}\}$ & $\{\mathrm{x}, \mathrm{z}, \mathrm{w}\}$ \\
& \\
\hline
\end{tabular}

6.

\begin{tabular}{|c|c|}
\hline $\mathrm{S}$ & $\psi(\mathrm{S})$ \\
\hline$\{\mathrm{x}, \mathrm{y}\}$ & $\{\mathrm{x}, \mathrm{y}, \mathrm{z}\}$ \\
$\{\mathrm{y}, \mathrm{z}\}$ & $\{\mathrm{y}, \mathrm{z}, \mathrm{w}\}$ \\
$\{\mathrm{x}, \mathrm{w}\}$ & $\{\mathrm{x}, \mathrm{w}, \mathrm{y}\}$ \\
$\{\mathrm{y}, \mathrm{w}\}$ & $\{\mathrm{y}, \mathrm{w}, \mathrm{z}\}$ \\
$\{\mathrm{z}, \mathrm{w}\}$ & $\{\mathrm{z}, \mathrm{w}, \mathrm{y}\}$ \\
$\{\mathrm{x}, \mathrm{z}\}$ & $\{\mathrm{x}, \mathrm{z}, \mathrm{w}\}$ \\
& \\
\hline
\end{tabular}

7.

\begin{tabular}{|c|c|}
\hline $\mathrm{S}$ & $\psi(S)$ \\
\hline$\{\mathrm{x}, \mathrm{y}\}$ & $\{\mathrm{x}, \mathrm{y}, \mathrm{z}\}$ \\
$\{\mathrm{y}, \mathrm{z}\}$ & $\{\mathrm{y}, \mathrm{z}, \mathrm{w}\}$ \\
$\{\mathrm{x}, \mathrm{w}\}$ & $\{\mathrm{x}, \mathrm{w}, \mathrm{z}\}$ \\
$\{\mathrm{y}, \mathrm{w}\}$ & $\{\mathrm{y}, \mathrm{w}, \mathrm{x}\}$ \\
$\{\mathrm{z}, \mathrm{w}\}$ & $\{\mathrm{z}, \mathrm{w}, \mathrm{x}\}$ \\
$\{\mathrm{x}, \mathrm{z}\}$ & $\{\mathrm{x}, \mathrm{y}, \mathrm{z}\}$ \\
& \\
\hline
\end{tabular}

8.

\begin{tabular}{|c|c|}
\hline $\mathrm{S}$ & $\psi(\mathrm{S})$ \\
\hline$\{\mathrm{x}, \mathrm{y}\}$ & $\{\mathrm{x}, \mathrm{y}, \mathrm{z}\}$ \\
$\{\mathrm{y}, \mathrm{z}\}$ & $\{\mathrm{y}, \mathrm{z}, \mathrm{w}\}$ \\
$\{\mathrm{x}, \mathrm{w}\}$ & $\{\mathrm{x}, \mathrm{w}, \mathrm{z}\}$ \\
$\{\mathrm{y}, \mathrm{w}\}$ & $\{\mathrm{y}, \mathrm{w}, \mathrm{x}\}$ \\
$\{\mathrm{z}, \mathrm{w}\}$ & $\{\mathrm{z}, \mathrm{w}, \mathrm{x}\}$ \\
$\{\mathrm{x}, \mathrm{z}\}$ & $\{\mathrm{x}, \mathrm{z}, \mathrm{w}\}$ \\
& \\
\hline
\end{tabular}




\begin{tabular}{|c|c|}
\hline $\mathrm{S}$ & $\psi(\mathrm{S})$ \\
\hline$\{\mathrm{x}, \mathrm{y}\}$ & $\{\mathrm{x}, \mathrm{y}, \mathrm{z}\}$ \\
$\{\mathrm{y}, \mathrm{z}\}$ & $\{\mathrm{y}, \mathrm{z}, \mathrm{w}\}$ \\
$\{\mathrm{x}, \mathrm{w}\}$ & $\{\mathrm{x}, \mathrm{w}, \mathrm{z}\}$ \\
$\{\mathrm{y}, \mathrm{w}\}$ & $\{\mathrm{y}, \mathrm{w}, \mathrm{x}\}$ \\
$\{\mathrm{z}, \mathrm{w}\}$ & $\{\mathrm{z}, \mathrm{w}, \mathrm{y}\}$ \\
$\{\mathrm{x}, \mathrm{z}\}$ & $\{\mathrm{x}, \mathrm{y}, \mathrm{z}\}$ \\
& \\
\hline
\end{tabular}

10.

\begin{tabular}{|c|c|}
\hline $\mathrm{S}$ & $\psi(S)$ \\
\hline$\{\mathrm{x}, \mathrm{y}\}$ & $\{\mathrm{x}, \mathrm{y}, \mathrm{z}\}$ \\
$\{\mathrm{y}, \mathrm{z}\}$ & $\{\mathrm{y}, \mathrm{z}, \mathrm{w}\}$ \\
$\{\mathrm{x}, \mathrm{w}\}$ & $\{\mathrm{x}, \mathrm{w}, \mathrm{z}\}$ \\
$\{\mathrm{y}, \mathrm{w}\}$ & $\{\mathrm{y}, \mathrm{w}, \mathrm{x}\}$ \\
$\{\mathrm{z}, \mathrm{w}\}$ & $\{\mathrm{z}, \mathrm{w}, \mathrm{y}\}$ \\
$\{\mathrm{x}, \mathrm{z}\}$ & $\{\mathrm{x}, \mathrm{z}, \mathrm{w}\}$ \\
& \\
\hline
\end{tabular}

These don't comprise ten genuinely distinct cases because some may be obtained from others just by permuting letters. If one relevance map is obtained from another just by permuting letters, then either both will allow rules that satisfy ISA, Pareto, full domain, transitivity, and anonymity or neither will. Most of the ten cases can be obtained from the first by permuting letters. For example, suppose we take map 1 ,

\begin{tabular}{|c|c|}
\hline $\mathrm{S}$ & $\psi(\mathrm{S})$ \\
\hline & \\
$\{\mathrm{x}, \mathrm{y}\}$ & $\{\mathrm{x}, \mathrm{y}, \mathrm{z}\}$ \\
$\{\mathrm{y}, \mathrm{z}\}$ & $\{\mathrm{y}, \mathrm{z}, \mathrm{w}\}$ \\
$\{\mathrm{x}, \mathrm{w}\}$ & $\{\mathrm{x}, \mathrm{w}, \mathrm{y}\}$ \\
$\{\mathrm{y}, \mathrm{w}\}$ & $\{\mathrm{y}, \mathrm{w}, \mathrm{x}\}$ \\
$\{\mathrm{z}, \mathrm{w}\}$ & $\{\mathrm{z}, \mathrm{w}, \mathrm{x}\}$ \\
$\{\mathrm{x}, \mathrm{z}\}$ & $\{\mathrm{x}, \mathrm{y}, \mathrm{z}\}$ \\
& \\
\hline
\end{tabular}

and replace w by $\mathrm{z}, \mathrm{x}$ by $\mathrm{w}, \mathrm{y}$ by $\mathrm{x}$, and $\mathrm{z}$ by $\mathrm{y}$. The result is 


\begin{tabular}{|c|c|}
\hline $\mathrm{S}$ & $\psi(\mathrm{S})$ \\
\hline$\{\mathrm{w}, \mathrm{x}\}$ & $\{\mathrm{w}, \mathrm{x}, \mathrm{y}\}$ \\
$\{\mathrm{x}, \mathrm{y}\}$ & $\{\mathrm{x}, \mathrm{y}, \mathrm{z}\}$ \\
$\{\mathrm{w}, \mathrm{z}\}$ & $\{\mathrm{w}, \mathrm{z}, \mathrm{x}\}$ \\
$\{\mathrm{x}, \mathrm{z}\}$ & $\{\mathrm{x}, \mathrm{z}, \mathrm{w}\}$ \\
$\{\mathrm{y}, \mathrm{z}\}$ & $\{\mathrm{y}, \mathrm{z}, \mathrm{w}\}$ \\
$\{\mathrm{w}, \mathrm{y}\}$ & $\{\mathrm{w}, \mathrm{x}, \mathrm{y}\}$ \\
& \\
\hline
\end{tabular}

which is the same as map 2:

\begin{tabular}{|c|c|}
\hline $\mathrm{S}$ & $\psi(\mathrm{S})$ \\
\hline$\{\mathrm{x}, \mathrm{y}\}$ & $\{\mathrm{x}, \mathrm{y}, \mathrm{z}\}$ \\
$\{\mathrm{y}, \mathrm{z}\}$ & $\{\mathrm{y}, \mathrm{z}, \mathrm{w}\}$ \\
$\{\mathrm{x}, \mathrm{w}\}$ & $\{\mathrm{x}, \mathrm{w}, \mathrm{y}\}$ \\
$\{\mathrm{y}, \mathrm{w}\}$ & $\{\mathrm{y}, \mathrm{w}, \mathrm{x}\}$ \\
$\{\mathrm{z}, \mathrm{w}\}$ & $\{\mathrm{z}, \mathrm{w}, \mathrm{x}\}$ \\
$\{\mathrm{x}, \mathrm{z}\}$ & $\{\mathrm{x}, \mathrm{z}, \mathrm{w}\}$ \\
& \\
\hline
\end{tabular}

The following table shows how maps $2,3,4,5,7,9$, and 10 can be derived from map 1 by permutations. The first component is the image of $\mathrm{w}$, the second is the image of $\mathrm{x}$, then $\mathrm{y}$, and then $\mathrm{z}$.

\begin{tabular}{|c|c|}
\hline Map & Permutation \\
\hline 2 & $(\mathrm{z}, \mathrm{w}, \mathrm{x}, \mathrm{y})$ \\
3 & $(\mathrm{z}, \mathrm{w}, \mathrm{y}, \mathrm{x})$ \\
4 & $(\mathrm{x}, \mathrm{y}, \mathrm{z}, \mathrm{w})$ \\
5 & $(\mathrm{y}, \mathrm{z}, \mathrm{w}, \mathrm{x})$ \\
7 & $(\mathrm{w}, \mathrm{x}, \mathrm{z}, \mathrm{y})$ \\
9 & $(\mathrm{x}, \mathrm{z}, \mathrm{y}, \mathrm{w})$ \\
10 & $(\mathrm{x}, \mathrm{z}, \mathrm{w}, \mathrm{y})$ \\
& \\
\hline
\end{tabular}

This leaves maps 6 and 8 . Neither can be derived from map 1 by permuting letters. To see this, note that for the eight cases above, all four letters occur as $\mathrm{c}$ in a map image

$$
\psi(\{a, b\})=\{a, b, c\}
$$


but in maps 6 and 8, only three letters occur this way. However, map 8 can be obtained from map 6 by the permutation $(\mathrm{x}, \mathrm{y}, \mathrm{z}, \mathrm{w})$. Therefore there are only two significantly different cases, \#1 and \#6. Neither admits a transitive-valued social welfare function on a full domain satisfying ISA, Pareto, and anonymity. We take the two maps up in turn.

\section{Relevance Map \#1}

We are treating the relevance map:

\begin{tabular}{|c|c|}
\hline $\mathrm{S}$ & $\psi(\mathrm{S})$ \\
\hline$\{\mathrm{x}, \mathrm{y}\}$ & $\{\mathrm{x}, \mathrm{y}, \mathrm{z}\}$ \\
$\{\mathrm{y}, \mathrm{z}\}$ & $\{\mathrm{y}, \mathrm{z}, \mathrm{w}\}$ \\
$\{\mathrm{x}, \mathrm{w}\}$ & $\{\mathrm{x}, \mathrm{w}, \mathrm{y}\}$ \\
$\{\mathrm{y}, \mathrm{w}\}$ & $\{\mathrm{y}, \mathrm{w}, \mathrm{x}\}$ \\
$\{\mathrm{z}, \mathrm{w}\}$ & $\{\mathrm{z}, \mathrm{w}, \mathrm{x}\}$ \\
$\{\mathrm{x}, \mathrm{z}\}$ & $\{\mathrm{x}, \mathrm{y}, \mathrm{z}\}$ \\
& \\
\hline
\end{tabular}

Since $\psi(\{x y\})=\{x, y, z\}$ and not $\{x, y\}$, there must be a pair of profiles that agree on $\{x, y\}$ but yield different social rankings on $\mathrm{x}, \mathrm{y}$ (because they don't agree on all of $\{\mathrm{x}, \mathrm{y}, \mathrm{z}\}$ ). By Pareto, these must be profiles on which the two individuals disagree on $\mathrm{x}$ and $\mathrm{y}$. There are nine kinds of profiles that must be considered, each described by a subprofile:

\begin{tabular}{|c|c|c|c|c|c|c|c|c|}
\hline $\mathrm{u}_{1}$ & $\mathrm{u}_{2}$ & $\mathrm{u}_{3}$ & $\mathrm{u}_{4}$ & $\mathrm{u}_{5}$ & $\mathrm{u}_{6}$ & $\mathrm{u}_{7}$ & $\mathrm{u}_{8}$ & $\mathrm{u}_{9}$ \\
\hline $\mathrm{z} \mathrm{z}$ & $\mathrm{z} \mathrm{y}$ & $\mathrm{z} \mathrm{y}$ & $\mathrm{x} \mathrm{z}$ & $\mathrm{x} \mathrm{y}$ & $\mathrm{x} \mathrm{y}$ & $\mathrm{x} \mathrm{z}$ & $\mathrm{x} \mathrm{y}$ & $\mathrm{x} \mathrm{y}$ \\
$\mathrm{x} \mathrm{y}$ & $\mathrm{x} \mathrm{z}$ & $\mathrm{x} \mathrm{x}$ & $\mathrm{z} \mathrm{y}$ & $\mathrm{z} \mathrm{z}$ & $\mathrm{z} \mathrm{x}$ & $\mathrm{y} \mathrm{y}$ & $\mathrm{y} \mathrm{z}$ & $\mathrm{y} \mathrm{x}$ \\
$\mathrm{y} \mathrm{x}$ & $\mathrm{y} \mathrm{x}$ & $\mathrm{y} \mathrm{z}$ & $\mathrm{y} \mathrm{x}$ & $\mathrm{y} \mathrm{x}$ & $\mathrm{y} \mathrm{z}$ & $\mathrm{z} \mathrm{x}$ & $\mathrm{z} \mathrm{x}$ & $\mathrm{z} \mathrm{z}$ \\
\hline
\end{tabular}

So for at least one of these subprofiles, $\mathrm{x} \geq \mathrm{y}$ must hold in the social ranking. Our first step is to show that we need only consider the sixth profile on the list.

Proposition 1: If $\mathrm{f}$ is a rule satisfying all of ISA, full domain, transitivity, and anonymity, then for each subprofile $\mathrm{u}_{\mathrm{i}}$ in the table, if $\mathrm{x} \geq \mathrm{y}$ at $\mathrm{u}_{\mathrm{i}}$, then we also have $\mathrm{x} \geq \mathrm{y}$ at $\mathrm{u}_{6}$.

Proof: The steps in this proof are much like the steps in the contagion part of the standard proof of Arrow's theorem. However, because the relevance map here is more complicated than that for a rule satisfying IIA, there are many more steps. We treat $\mathrm{u}_{1}$ explicitly.

The nine sub-profiles are identical when restricted to $\{\mathrm{x}, \mathrm{y}\}$. So for any $\mathrm{u}_{\mathrm{i}}$ we merely insert $\mathrm{w}$ just below $\mathrm{y}$ to get $\mathrm{y} \succ \mathrm{w}$ 
and thus the conclusion of (2) by transitivity. The remaining steps apply without modification, resulting in $\mathrm{x} \succ \mathrm{y}$ at $\mathrm{u}_{6}$.

Assume $\mathrm{x} \succeq \mathrm{y}$ in the social ranking at $\mathrm{u}_{1}$ :

(1)

\section{ZZ}

$\mathrm{xy} \rightarrow \mathrm{x} \geq \mathrm{y}$.

yx

and then establish a sequence of deductions ${ }^{2}$ :

(2)

\begin{tabular}{|l|l|l|l|l|}
\hline zz & & & & \\
xy & $\mathrm{x} \geq \mathrm{y}$ & $\mathrm{y} \succ \mathrm{w}$ & $\mathrm{x} \succ \mathrm{w}$ & $\mathrm{xy}$ \\
$\mathrm{yw}$ & by (1) & by(P) & & \\
$\mathrm{wx}$ & & & & \\
& & & & \\
\hline
\end{tabular}

(3)

\begin{tabular}{|l|l|l|l|l|}
\hline zy & & & \\
xw & $\mathrm{x} \succ \mathrm{w}$ & $\mathrm{z} \succ \mathrm{x}$ & $\mathrm{zw}$ \\
$\mathrm{yz}$ & by (2) & by (P) & $\mathrm{z} \succ \mathrm{w}$ & $\mathrm{xz} \rightarrow \mathrm{z} \succ \mathrm{w}$ \\
$\mathrm{wx}$ & & & & \\
\hline
\end{tabular}

(4)

\begin{tabular}{|l|l|l|l|l|}
\hline zw & & & \\
xy & $\mathrm{z} \succ \mathrm{w}$ & $\mathrm{w} \succ \mathrm{y}$ & $\mathrm{z} \succ \mathrm{y}$ & $\mathrm{zy} \rightarrow \mathrm{z} \succ \mathrm{y}$ \\
$\mathrm{wz}$ & by (3) & by (P) & & \\
$\mathrm{yx}$ & & & & \\
\hline
\end{tabular}

2 In tables like this, the first cell contains a profile being analyzed. The second and third cells are partial descriptions of the value at the profile for any rule satisfying all of: ISA, Pareto, full domain, transitivity, and anonymity. [Note: "by P" means by the Pareto condition.] The fourth cell is a transitivity consequence of the entries in cells two and three. The last cell is an implication derived from the relevance map, the profile in the first cell and the consequence in the fourth cell. 
(5)

\begin{tabular}{|l|c|c|l|l|}
\hline xw & & & & \\
zy & $\mathrm{z} \succ \mathrm{y}$ & $\mathrm{x}>\mathrm{z}$ & $\mathrm{xy}$ \\
$\mathrm{wx}$ & by (4) & by (P) & $\mathrm{x} \succ \mathrm{y}$ & $\mathrm{zx} \rightarrow \mathrm{x} \succ \mathrm{y}$ \\
$\mathrm{yz}$ & & & $\mathrm{yz}$ \\
\hline
\end{tabular}

This last conclusion is what we sought: $\mathrm{x} \succ \mathrm{y}$ in the social ranking at subprofile $\mathrm{u}_{6}$.

Notice that Proposition 1 did not use anonymity.

It now suffices to prove that, for the sixth subprofile in the table there is no rule satisfying Pareto, full domain, transitivity, and anonymity for which the relevance map $\psi$ is that of case \#1 and where $\mathrm{x} \geq \mathrm{y}$ at subprofile $\mathrm{u}_{6}$. During that proof we will need to know that a similar claim is true for having $\mathrm{x} \succeq \mathrm{y}$ at subprofile $\mathrm{u}_{1}$, so we begin there.

\section{First subprofile.}

Proposition 2: There is no rule satisfying all of ISA, Pareto, full domain, transitivity, and anonymity with relevance map \#1 such that

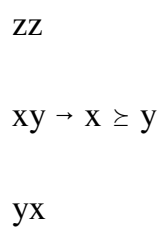

Proof: We are going to show inconsistency by a sequence of deductions that start from

(1)

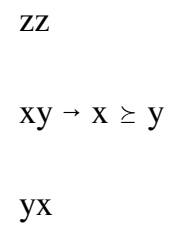

Our first goal will be to prove

$\mathrm{XW}$

$$
\mathrm{yx} \rightarrow \mathrm{w} \succ \mathrm{y}
$$

wy $\mathrm{xz}$

$$
\mathrm{zy} \rightarrow \mathrm{z} \succ \mathrm{x}
$$

yx

although we will use some of the steps in the proof at a later stage. 
(2)

\begin{tabular}{|l|c|c|l|l|}
\hline wz & & & & \\
zy & $\mathrm{x} \geq \mathrm{y}$ & $\mathrm{w} \succ \mathrm{x}$ & $\mathrm{wy}$ \\
$\mathrm{xw}$ & by (1) & by (P) & $\mathrm{w} \succ \mathrm{y}$ & $\mathrm{xw} \rightarrow \mathrm{w} \succ \mathrm{y}$ \\
$\mathrm{yx}$ & & & $\mathrm{yx}$ \\
\hline
\end{tabular}

(3)

\begin{tabular}{|l|c|c|l|l|}
\hline wy & & & & \\
xz & w $>\mathrm{y}$ & $\mathrm{y}>\mathrm{z}$ & $\mathrm{wz}$ \\
$\mathrm{yw}$ & by (2) & by (P) & $\mathrm{w} \succ \mathrm{z}$ & $\mathrm{xw} \rightarrow \mathrm{w} \succ \mathrm{z}$ \\
$\mathrm{zx}$ & & & $\mathrm{zx}$ \\
\hline
\end{tabular}

(4)

\begin{tabular}{|l|l|l|l|l|}
\hline yz & & & & \\
wy & $\mathrm{w}>\mathrm{z}$ & $\mathrm{y} \succ \mathrm{w}$ & $\mathrm{yz}$ \\
$\mathrm{xw}$ & by (3) & by (P) & $\mathrm{y} \succ \mathrm{z}$ & $\mathrm{wy} \rightarrow \mathrm{y}>\mathrm{z}$ \\
$\mathrm{zx}$ & & & $\mathrm{zw}$ \\
\hline
\end{tabular}

(5)

\begin{tabular}{|l|c|c|l|l|}
\hline yz & & & & \\
wx & $y>z$ & $z \succ x$ & $y z$ \\
$z y$ & by (4) & by (P) & $y>x$ & $\mathrm{zx} \rightarrow \mathrm{y} \succ \mathrm{x}$ \\
$\mathrm{xw}$ & & & & \\
\hline
\end{tabular}


(6)

\begin{tabular}{|l|c|c|l|l|}
\hline zw & & & & \\
xy & y $>\mathrm{x}$ & $\mathrm{w} \succ \mathrm{y}$ & & $\mathrm{xw}$ \\
$\mathrm{wz}$ & by $\left(5^{*}\right)$ & by (P) & $\mathrm{w} \succ \mathrm{x}$ & $\mathrm{wy} \rightarrow \mathrm{w} \succ \mathrm{x}$ \\
$\mathrm{yx}$ & & & $\mathrm{yx}$ \\
\hline
\end{tabular}

This derivation of (6) from (5) uses anonymity; we indicate this by the asterisk in (5*).

Henceforth, we will use asterisks to indicate applications of anonymity.

(7)

\begin{tabular}{|l|c|c|l|l|}
\hline $\mathrm{xw}$ & & & & \\
$\mathrm{zy}$ & $\mathrm{w} \succ \mathrm{x}$ & $\mathrm{x} \succ \mathrm{z}$ & $\mathrm{xw}$ \\
$\mathrm{wx}$ & $\mathrm{by}(6)$ & $\mathrm{by}(\mathrm{P})$ & $\mathrm{w} \succ \mathrm{z}$ & $\mathrm{zx} \rightarrow \mathrm{w} \succ \mathrm{z}$ \\
$\mathrm{yz}$ & & & & \\
\hline
\end{tabular}

(8)

\begin{tabular}{|l|l|l|l|l|}
\hline $\mathrm{xw}$ & & & & \\
$\mathrm{zx}$ & $\mathrm{w} \succ \mathrm{z}$ & $\mathrm{z} \succ \mathrm{y}$ & & $\mathrm{xw}$ \\
$\mathrm{yz}$ & by (7) & by (P) & $\mathrm{w} \succ \mathrm{y}$ & $\mathrm{y}>\mathrm{y}$ \\
& & & \\
\hline
\end{tabular}

(9)

\begin{tabular}{|l|l|l|l|l|}
\hline zz & & & & \\
$\mathrm{xy}$ & $\mathrm{x} \geq \mathrm{y}$ & $\mathrm{y} \succ \mathrm{w}$ & $\mathrm{xy}$ \\
$\mathrm{yw}$ & by (1) & by (P) & $\mathrm{x} \succ \mathrm{w}$ & $\mathrm{yw} \rightarrow \mathrm{x} \succ \mathrm{w}$ \\
$\mathrm{wx}$ & & & & \\
\hline
\end{tabular}


(10)

\begin{tabular}{|l|l|l|l|l|}
\hline $\mathrm{xz}$ & & & & \\
$\mathrm{zw}$ & $\mathrm{w} \succ \mathrm{x}$ & $\mathrm{z} \succ \mathrm{w}$ & $\mathrm{xz}$ \\
wy & by (6) & by (P) & $\mathrm{z} \succ \mathrm{x}$ & $\mathrm{zy} \rightarrow \mathrm{z} \succ \mathrm{x}$ \\
$\mathrm{yx}$ & & & $\mathrm{yx}$ \\
\hline
\end{tabular}

Our second goal will be to prove two more results:

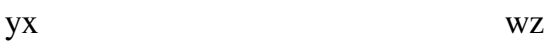
(13)
$\mathrm{zy} \rightarrow \mathrm{x} \succ \mathrm{z}$
(14)
$\mathrm{zX} \rightarrow \mathrm{z} \succ \mathrm{W}$
$\mathrm{xz}$
$\mathrm{xW}$

although again we will use later some of the steps in the sequence to these results.

(11)

\begin{tabular}{|l|l|l|l|l|}
\hline zy & & & & \\
wz & w $\geq \mathrm{y}$ & $\mathrm{z} \succ \mathrm{w}$ & $\mathrm{zy}$ \\
$\mathrm{xw}$ & by (2) & by (P) & $\mathrm{z} \succ \mathrm{y}$ & $\mathrm{wz} \rightarrow \mathrm{z} \succ \mathrm{y}$ \\
$\mathrm{yx}$ & & & $\mathrm{yw}$ \\
\hline
\end{tabular}

(12)

\begin{tabular}{|l|c|c|l|l|}
\hline yx & & & & \\
$\mathrm{xz}$ & $\mathrm{z}>\mathrm{y}$ & $\mathrm{x}>\mathrm{z}$ & $\mathrm{yx}$ \\
$\mathrm{zw}$ & by $\left(11^{*}\right)$ & by (P) & $\mathrm{x} \succ \mathrm{y}$ & $\mathrm{xz} \rightarrow \mathrm{x}>\mathrm{y}$ \\
wy & & & $\mathrm{zy}$ \\
\hline
\end{tabular}


(13)

\begin{tabular}{|l|c|c|l|l|}
\hline yx & & & & \\
wy & $\mathrm{x} \geq \mathrm{w}$ & $\mathrm{w} \succ \mathrm{z}$ & $\mathrm{yx}$ \\
$\mathrm{zw}$ & by $(9 *)$ & by (P) & $\mathrm{x} \succ \mathrm{z}$ & $\mathrm{zy} \rightarrow \mathrm{x}>\mathrm{z}$ \\
$\mathrm{xz}$ & & & $\mathrm{xz}$ \\
\hline
\end{tabular}

(14)

\begin{tabular}{|l|c|c|l|l|}
\hline yz & & & & \\
wx & $\mathrm{x}>\mathrm{w}$ & $\mathrm{z} \succ \mathrm{x}$ & $\mathrm{wz}$ \\
$\mathrm{zy}$ & by (9*) & by (P) & $\mathrm{z} \succ \mathrm{w}$ & $\mathrm{zx} \rightarrow \mathrm{z} \succ \mathrm{w}$ \\
$\mathrm{xw}$ & & & \\
\hline
\end{tabular}

At this point, our derivations split into two paths depending on the social ordering of $\mathrm{x}$ and $\mathrm{z}$ at the subprofile

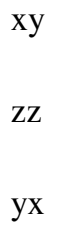

In the first path we assume $\mathrm{x} \succ \mathrm{z}$ and in the second we assume $\mathrm{z} \geq \mathrm{x}$. At the end of each path we will find a contradiction.

\section{First path}

xy

$$
\mathrm{zZ} \rightarrow \mathrm{x}>\mathrm{Z}
$$

yx

(16)

\begin{tabular}{|l|c|c|l|l|}
\hline xy & & & & \\
zz & $\mathrm{x} \succ \mathrm{z}$ & $\mathrm{z} \succ \mathrm{w}$ & $\mathrm{xy}$ \\
$\mathrm{ww}$ & by (15) & by (P) & $\mathrm{x} \succ \mathrm{w}$ & $\mathrm{ww} \rightarrow \mathrm{x} \succ \mathrm{w}$ \\
$\mathrm{yx}$ & & & $\mathrm{yx}$ \\
\hline
\end{tabular}


(17)

\begin{tabular}{|l|c|c|l|l|}
\hline zx & & & & \\
$\mathrm{yw}$ & $\mathrm{z} \succ \mathrm{x}$ & $\mathrm{x} \succ \mathrm{w}$ & $\mathrm{zx}$ \\
$\mathrm{wz}$ & by $\left(10^{*}\right)$ & by $\left(16^{*}\right)$ & $\mathrm{z} \succ \mathrm{w}$ & $\mathrm{ww} \rightarrow \mathrm{z} \succ \mathrm{w}$ \\
$\mathrm{xy}$ & & & $\mathrm{xz}$ \\
\hline
\end{tabular}

(18)

\begin{tabular}{|c|c|c|c|c|}
\hline $\begin{array}{l}\mathrm{zx} \\
\mathrm{wy} \\
\mathrm{xw} \\
\mathrm{yz}\end{array}$ & $\begin{array}{c}\text { w } \succ y \\
\text { by }\left(8^{*}\right)\end{array}$ & $\begin{array}{c}\mathrm{z} \succ \mathrm{w} \\
\text { by (17) }\end{array}$ & $\mathrm{z} \succ \mathrm{y}$ & $\begin{array}{l}\mathrm{zy} \\
\mathrm{ww} \rightarrow \mathrm{z} \succ \mathrm{y} \\
\mathrm{yz}\end{array}$ \\
\hline
\end{tabular}

But this result is contradicted by the conclusion of the following short sequence:

(19)

\begin{tabular}{|c|c|c|l|l|}
\hline yx & & & & \\
zw & $\mathrm{z} \succ w$ & $\mathrm{y} \succ \mathrm{z}$ & $\mathrm{yx}$ & \\
wy & by (17) & by (P) & $\mathrm{yw} \rightarrow \mathrm{w}>\mathrm{w}$ & $\mathrm{xy}$ \\
$\mathrm{xz}$ & & & & \\
\hline
\end{tabular}

(20)

\begin{tabular}{|c|c|c|l|l|}
\hline xy & & & & \\
zw & $y>w$ & $w>z$ & $y$ & \\
wx & by $\left(19^{*}\right)$ & by (7) & y $>\mathrm{z}$ & $\mathrm{ww} \rightarrow \mathrm{y}>\mathrm{z}$ \\
$\mathrm{yz}$ & & & $\mathrm{yz}$ \\
\hline
\end{tabular}


Second Path

(21)

xy

$\mathrm{zZ} \rightarrow \mathrm{Z} \geq \mathrm{X}$

yx

(22)

\begin{tabular}{|l|c|l|l|l|}
\hline xy & & & & \\
zw & $\mathrm{z} \geq \mathrm{x}$ & $\mathrm{x} \succ \mathrm{w}$ & $\mathrm{xw}$ \\
$\mathrm{yz}$ & by (21) & by (9) & $\mathrm{z} \succ \mathrm{w}$ & $\mathrm{zz} \rightarrow \mathrm{z} \succ \mathrm{w}$ \\
$\mathrm{wx}$ & & & & \\
\hline
\end{tabular}

(23)

\begin{tabular}{|c|c|c|c|c|}
\hline $\begin{array}{l}\text { xy } \\
\text { ww } \\
z z \\
\text { yx }\end{array}$ & $\begin{array}{c}\mathrm{z} \succeq \mathrm{x} \\
\text { by }(21)\end{array}$ & $\begin{array}{l}\text { w } \succ \mathrm{z} \\
\text { by }(\mathrm{P})\end{array}$ & $\mathrm{w} \succ \mathrm{x}$ & $\begin{array}{l}\mathrm{xy} \\
\mathrm{ww} \rightarrow \mathrm{w} \succ \mathrm{x} \\
\mathrm{yx}\end{array}$ \\
\hline
\end{tabular}

(24)

\begin{tabular}{|l|c|c|l|l|}
\hline xy & & & \\
zw & w $>\mathrm{x}$ & $\mathrm{x}>\mathrm{y}$ & $\mathrm{xy}$ \\
$\mathrm{wx}$ & by (23) & by $\left(12^{*}\right)$ & $\mathrm{w} \succ \mathrm{y}$ & $\mathrm{ww} \rightarrow \mathrm{w} \succ \mathrm{y}$ \\
$\mathrm{yz}$ & & & $\mathrm{yx}$ \\
\hline
\end{tabular}


(25)

\begin{tabular}{|l|c|c|l|l|}
\hline zy & & & & \\
$\mathrm{xw}$ & $\mathrm{w} \succ \mathrm{y}$ & $\mathrm{z} \succ \mathrm{w}$ & $\mathrm{zy}$ \\
$\mathrm{wz}$ & by (24) & by $\left(14^{*}\right)$ & $\mathrm{z} \succ \mathrm{y}$ & $\mathrm{ww} \rightarrow \mathrm{z} \succ \mathrm{y}$ \\
$\mathrm{yx}$ & & & $\mathrm{yz}$ \\
\hline
\end{tabular}

But this result is contradicted by the conclusion of the following short sequence (and an application of anonymity):

(26)

\begin{tabular}{|l|c|c|l|l|}
\hline xw & & & & \\
yy & $\mathrm{z}>\mathrm{w}$ & $\mathrm{y}>\mathrm{z}$ & & $\mathrm{xw}$ \\
$\mathrm{zz}$ & by (22) & by (P) & $\mathrm{y} \succ \mathrm{w}$ & $\mathrm{yy} \rightarrow \mathrm{y}>\mathrm{w}$ \\
$\mathrm{wx}$ & & & $\mathrm{wx}$ \\
\hline
\end{tabular}

(27)

\begin{tabular}{|l|c|c|l|l|}
\hline xy & & & & \\
wz & w $>\mathrm{x}$ & $\mathrm{x}>\mathrm{z}$ & $\mathrm{xz}$ \\
$\mathrm{yw}$ & by (23) & by $\left(13^{*}\right)$ & $\mathrm{w} \succ \mathrm{z}$ & $\mathrm{ww} \rightarrow \mathrm{w}>\mathrm{z}$ \\
$\mathrm{zx}$ & & & $\mathrm{zx}$ \\
\hline
\end{tabular}

(28)

\begin{tabular}{|c|c|c|c|c|}
\hline $\begin{array}{l}\mathrm{xz} \\
\mathrm{yw} \\
\text { wy } \\
\mathrm{zx}\end{array}$ & $\begin{array}{c}\text { w } \succ \mathrm{z} \\
\text { by (27) }\end{array}$ & $\begin{array}{c}\mathrm{y} \succ \mathrm{w} \\
\text { by }(26)\end{array}$ & $\mathrm{y} \succ \mathrm{z}$ & $\begin{array}{l}\mathrm{yz} \\
\mathrm{ww} \rightarrow \mathrm{y} \succ \mathrm{z} \\
\mathrm{zy}\end{array}$ \\
\hline
\end{tabular}




\section{Sixth subprofile.}

Proposition 3: There is no rule satisfying all of ISA, Pareto, full domain, transitivity, and anonymity with relevance map \#1 such that

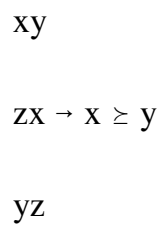

Proof: This proof is more complicated than the preceding ones. Of course we start by assuming

(1)

$$
\begin{aligned}
& x y \\
& z x \rightarrow x \geq y . \\
& y z
\end{aligned}
$$

and then derive a contradiction.

(2)

\begin{tabular}{|l|c|c|l|l|}
\hline wy & & & & \\
$\mathrm{xw}$ & $\mathrm{x} \geq \mathrm{y}$ & $\mathrm{w} \succ \mathrm{x}$ & $\mathrm{wy}$ \\
$\mathrm{zx}$ & by (1) & by (P) & $\mathrm{w} \succ \mathrm{y}$ & $\mathrm{xw} \rightarrow \mathrm{w} \succ \mathrm{y}$ \\
$\mathrm{yz}$ & & & $\mathrm{yx}$ \\
\hline
\end{tabular}

(3)

\begin{tabular}{|l|c|c|l|l|}
\hline $\mathrm{xy}$ & & & & \\
$\mathrm{zw}$ & $\mathrm{x} \geq \mathrm{y}$ & $\mathrm{y} \succ \mathrm{w}$ & & $\mathrm{xy}$ \\
$\mathrm{yx}$ & by (1) & by (P) & $\mathrm{x} \succ \mathrm{w}$ & $\mathrm{yw} \rightarrow \mathrm{x} \succ \mathrm{w}$ \\
$\mathrm{wz}$ & & & & \\
\hline
\end{tabular}


(4)

\begin{tabular}{|l|l|l|l|l|}
\hline zy & & & & \\
wz & w $>\mathrm{y}$ & $\mathrm{z} \succ \mathrm{w}$ & $\mathrm{zy}$ \\
$\mathrm{xw}$ & by (2) & by (P) & $\mathrm{z} \succ \mathrm{y}$ & $\mathrm{wz} \rightarrow \mathrm{z} \succ \mathrm{y}$ \\
$\mathrm{yx}$ & & & $\mathrm{yw}$ \\
\hline
\end{tabular}

(5)

\begin{tabular}{|l|c|c|l|l|}
\hline wy & & & & \\
$\mathrm{xz}$ & $\mathrm{w}>\mathrm{y}$ & $\mathrm{y}>\mathrm{z}$ & & $\mathrm{wz}$ \\
$\mathrm{yw}$ & by (2) & by (P) & $\mathrm{w} \succ \mathrm{z}$ & $\mathrm{xw} \rightarrow \mathrm{w} \succ \mathrm{z}$ \\
$\mathrm{zx}$ & & & $\mathrm{zx}$ \\
\hline
\end{tabular}

(6)

\begin{tabular}{|l|l|l|l|l|}
\hline yz & & & & \\
wy & w $>\mathrm{z}$ & $\mathrm{y} \succ \mathrm{w}$ & & $\mathrm{yz}$ \\
$\mathrm{xw}$ & by (5) & by (P) & $\mathrm{y} \succ \mathrm{z}$ & $\mathrm{wy} \rightarrow \mathrm{y}>\mathrm{z}$ \\
$\mathrm{zx}$ & & & $\mathrm{zw}$ \\
\hline
\end{tabular}

(7)

\begin{tabular}{|l|c|c|l|l|}
\hline zy & & & & \\
wx & $\mathrm{z} \succ \mathrm{y}$ & $\mathrm{y} \succ \mathrm{x}$ & $\mathrm{zy}$ \\
$\mathrm{yz}$ & by (4) & by (P) & $\mathrm{z} \succ \mathrm{x}$ & $\mathrm{yx} \rightarrow \mathrm{z} \succ \mathrm{x}$ \\
$\mathrm{xw}$ & & & & \\
\hline
\end{tabular}


(8)

\begin{tabular}{|l|c|c|l|l|}
\hline wy & & & & \\
$\mathrm{zx}$ & $\mathrm{z} \succ \mathrm{x}$ & $\mathrm{w} \succ \mathrm{z}$ & & $\mathrm{wy}$ \\
$\mathrm{yw}$ & by (7) & by (P) & $\mathrm{w} \succ \mathrm{x}$ & $\mathrm{yx}>\mathrm{w}>\mathrm{x}$ \\
$\mathrm{xz}$ & & & & \\
\hline
\end{tabular}

(9)

\begin{tabular}{|l|c|c|l|l|}
\hline wy & & & & \\
$\mathrm{yx}$ & $\mathrm{w}>\mathrm{x}$ & $\mathrm{x}>\mathrm{z}$ & & $\mathrm{wx}$ \\
$\mathrm{xz}$ & by (8) & by (P) & $\mathrm{w} \succ \mathrm{z}$ & $\mathrm{xz} \rightarrow \mathrm{w}>\mathrm{z}$ \\
$\mathrm{zw}$ & & & $\mathrm{zw}$ \\
\hline
\end{tabular}

(10)

\begin{tabular}{|l|c|c|l|l|}
\hline wx & & & & \\
$\mathrm{xz}$ & $\mathrm{w} \succ \mathrm{z}$ & $\mathrm{z} \succ \mathrm{y}$ & & $\mathrm{wx}$ \\
$\mathrm{zy}$ & by (9) & by (P) & $\mathrm{w} \succ \mathrm{y}$ & $\mathrm{xy}>\mathrm{w} \succ \mathrm{y}$ \\
$\mathrm{yw}$ & & & & \\
\hline
\end{tabular}

(11)

\begin{tabular}{|l|c|c|l|l|}
\hline yz & & & & \\
wx & $y>z$ & $z \succ x$ & $y z$ & \\
zy & by (6) & by (P) & $y>x$ & zx $\rightarrow y \succ x$ \\
xw & & & & \\
\hline
\end{tabular}


(12)

\begin{tabular}{|l|c|c|l|l|}
\hline wz & & & & \\
$\mathrm{yx}$ & $\mathrm{y}>\mathrm{x}$ & $\mathrm{w}>\mathrm{y}$ & & $\mathrm{wx}$ \\
$\mathrm{zw}$ & by (11) & by (P) & $\mathrm{w} \succ \mathrm{x}$ & $\mathrm{yw}>\mathrm{w}$ \\
$\mathrm{xy}$ & & & & \\
\hline
\end{tabular}

We now interrupt this sequence to introduce two new claims. The first is

(13)

$$
\begin{aligned}
& z z \\
& x y \rightarrow y \succ x . \\
& y x
\end{aligned}
$$

This, of course, is the first subprofile and we showed earlier that $\mathrm{x} \geq \mathrm{y}$ led to a contradiction. The second claim is

$$
\begin{aligned}
& z x \\
& y y \rightarrow y>x . \\
& x z
\end{aligned}
$$

We will prove (14) by assuming the contrary

$$
\mathrm{zx}
$$$$
\mathrm{yy} \rightarrow \mathrm{x} \geq \mathrm{y}
$$

$\mathrm{xZ}$

and showing that leads to a contradiction.

(16)

\begin{tabular}{|l|c|c|l|l|}
\hline zx & & & & \\
yy & $\mathrm{x} \geq \mathrm{y}$ & $\mathrm{y}>\mathrm{z}$ & $\mathrm{zx}$ \\
$\mathrm{xw}$ & by (15) & by $\left(6^{*}\right)$ & $\mathrm{x} \succ \mathrm{z}$ & $\mathrm{yy} \rightarrow \mathrm{x}>\mathrm{z}$ \\
$\mathrm{wz}$ & & & $\mathrm{xz}$ \\
\hline
\end{tabular}


(17)

\begin{tabular}{|l|c|c|l|l|}
\hline xz & & & & \\
yw & $\mathrm{x} \succ \mathrm{z}$ & $\mathrm{z} \succ \mathrm{w}$ & & $\mathrm{xw}$ \\
$\mathrm{zy}$ & by $\left(16^{*}\right)$ & by (P) & $\mathrm{x} \succ \mathrm{w}$ & $\mathrm{yy} \rightarrow \mathrm{x} \succ \mathrm{w}$ \\
$\mathrm{wx}$ & & & & \\
\hline
\end{tabular}

(18)

\begin{tabular}{|l|c|c|l|l|}
\hline zz & & & & \\
xw & $\mathrm{x}>\mathrm{w}$ & $\mathrm{y}>\mathrm{x}$ & & $\mathrm{xw}$ \\
yy & by (17) & by (13) & $\mathrm{y} \succ \mathrm{w}$ & $\mathrm{yy}>\mathrm{y} \succ \mathrm{w}$ \\
$\mathrm{wx}$ & & & & \\
\hline
\end{tabular}

(19)

\begin{tabular}{|l|c|c|l|l|}
\hline wx & & & & \\
$\mathrm{yz}$ & $\mathrm{y} \succ \mathrm{w}$ & $\mathrm{w} \succ \mathrm{z}$ & $\mathrm{wz}$ \\
$\mathrm{xy}$ & by $\left(18^{*}\right)$ & by $\left(9^{*}\right)$ & $\mathrm{y} \succ \mathrm{z}$ & $\mathrm{yy} \rightarrow \mathrm{y} \succ \mathrm{z}$ \\
$\mathrm{zw}$ & & & $\mathrm{zw}$ \\
\hline
\end{tabular}

(20)

\begin{tabular}{|l|c|c|l|l|}
\hline wz & & & & \\
$\mathrm{xx}$ & $\mathrm{y} \succ \mathrm{z}$ & $\mathrm{w} \succ \mathrm{y}$ & $\mathrm{wz}$ \\
$\mathrm{yy}$ & by (19) & by (10) & $\mathrm{w} \succ \mathrm{z}$ & $\mathrm{xx} \rightarrow \mathrm{w} \succ \mathrm{z}$ \\
$\mathrm{zw}$ & & & $\mathrm{zw}$ \\
\hline
\end{tabular}


(21)

\begin{tabular}{|l|c|c|l|l|}
\hline yz & & & & \\
wx & w $\succ \mathrm{z}$ & $\mathrm{x} \succ \mathrm{w}$ & $\mathrm{yz}$ \\
$\mathrm{xy}$ & by (20) & by (3*) & $\mathrm{x} \succ \mathrm{z}$ & $\mathrm{xx} \rightarrow \mathrm{x} \succ \mathrm{z}$ \\
$\mathrm{zw}$ & & & $\mathrm{zy}$ \\
\hline
\end{tabular}

(22)

\begin{tabular}{|l|c|c|l|l|}
\hline yz & & & & \\
$\mathrm{xw}$ & $\mathrm{x} \succ \mathrm{z}$ & $\mathrm{z} \succ \mathrm{w}$ & & $\mathrm{yw}$ \\
$\mathrm{zx}$ & by (21) & by (P) & $\mathrm{x} \succ \mathrm{w}$ & $\mathrm{xx} \rightarrow \mathrm{x} \succ \mathrm{w}$ \\
wy & & & wy \\
\hline
\end{tabular}

The contradiction we seek will be between this conclusion of (22) and the result of a second sequence we now start:

(23)

\begin{tabular}{|l|c|c|l|l|}
\hline zx & & & & \\
wz & w $\succ x$ & & & \\
yw & by (12) & by (P) & $\mathrm{z} \succ \mathrm{x}$ & $\mathrm{yz} \rightarrow \mathrm{z} \succ \mathrm{x}$ \\
$\mathrm{xy}$ & & & $\mathrm{xy}$ \\
\hline
\end{tabular}

(24)

\begin{tabular}{|l|c|c|l|l|}
\hline $\mathrm{xw}$ & & & \\
$\mathrm{zz}$ & $\mathrm{x} \succ \mathrm{w}$ & $\mathrm{z} \succ \mathrm{x}$ & $\mathrm{xw}$ \\
$\mathrm{yy}$ & by (17) & by $\left(23^{*}\right)$ & $\mathrm{z} \succ \mathrm{w}$ & $\mathrm{zz} \rightarrow \mathrm{z} \succ \mathrm{w}$ \\
$\mathrm{wx}$ & & & $\mathrm{wx}$ \\
\hline
\end{tabular}


(25)

\begin{tabular}{|l|c|c|l|l|}
\hline $\mathrm{xw}$ & & & \\
$\mathrm{zy}$ & $\mathrm{z} \succ \mathrm{w}$ & $\mathrm{w} \succ \mathrm{x}$ & $\mathrm{z} \succ \mathrm{x}$ & $\mathrm{xy}$ \\
$\mathrm{wz}$ & by (24) & by $\left(12^{*}\right)$ & & $\mathrm{zz} \rightarrow \mathrm{z} \succ \mathrm{x}$ \\
$\mathrm{yx}$ & & & $\mathrm{yx}$ \\
\hline
\end{tabular}

(26)

\begin{tabular}{|l|c|c|l|l|}
\hline wy & & & & \\
$\mathrm{xz}$ & $\mathrm{w}>\mathrm{z}$ & $\mathrm{z} \succ \mathrm{x}$ & & $\mathrm{wy}$ \\
$\mathrm{zx}$ & by (20) & by (25) & $\mathrm{w} \succ \mathrm{x}$ & $\mathrm{xx} \rightarrow \mathrm{w} \succ \mathrm{x}$ \\
$\mathrm{yw}$ & & & $\mathrm{yw}$ \\
\hline
\end{tabular}

(26) and anonymity contradict (22); we were led to this by assuming (15) which must then be false, so (14) is true. We now return to our main line of derivations.

(27)

\begin{tabular}{|l|c|c|l|l|}
\hline zx & & & & \\
wy & $y>x$ & $z \succ y$ & & zx \\
yz & by (14) & by (4) & $\mathrm{z} \succ x$ & $\mathrm{yy} \rightarrow \mathrm{z} \succ \mathrm{x}$ \\
$\mathrm{xw}$ & & & $\mathrm{xz}$ \\
\hline
\end{tabular}

(28)

\begin{tabular}{|l|c|c|l|l|}
\hline xz & & & & \\
yy & $\mathrm{z} \succ \mathrm{x}$ & $\mathrm{x} \succ \mathrm{w}$ & $\mathrm{xz}$ \\
ww & by $\left(27^{*}\right)$ & by (3) & $\mathrm{z} \succ \mathrm{w}$ & $\mathrm{ww} \rightarrow \mathrm{z} \succ \mathrm{w}$ \\
$\mathrm{zx}$ & & & $\mathrm{zx}$ \\
\hline
\end{tabular}


(29)

\begin{tabular}{|l|c|c|l|l|}
\hline xy & & & & \\
wz & $\mathrm{z} \succ w$ & $\mathrm{y} \succ \mathrm{z}$ & & $\mathrm{xy}$ \\
$\mathrm{yw}$ & by (28) & by (P) & $\mathrm{y} \succ \mathrm{w}$ & $\mathrm{ww}>\mathrm{y} \succ \mathrm{w}$ \\
$\mathrm{zx}$ & & & & \\
\hline
\end{tabular}

The last contradiction we seek will be between this conclusion of (29) and the result of a short final sequence:

\begin{tabular}{|l|c|c|l|l|}
\hline $\mathrm{xz}$ & & & & \\
$\mathrm{yw}$ & $\mathrm{z} \succ \mathrm{w}$ & $\mathrm{w} \succ \mathrm{y}$ & $\mathrm{yz}$ \\
$\mathrm{wx}$ & by (28) & by $\left(10^{*}\right)$ & $\mathrm{z} \succ \mathrm{y}$ & $\mathrm{ww} \rightarrow \mathrm{z} \succ \mathrm{y}$ \\
$\mathrm{zy}$ & & & $\mathrm{zy}$ \\
\hline
\end{tabular}

(31)

\begin{tabular}{|l|c|c|l|l|}
\hline yx & & & & \\
wz & $\mathrm{z} \succ \mathrm{y}$ & $\mathrm{w} \succ \mathrm{z}$ & & $\mathrm{yx}$ \\
$\mathrm{xw}$ & by (30) & by (9) & $\mathrm{w} \succ \mathrm{y}$ & $\mathrm{ww} \rightarrow \mathrm{w} \succ \mathrm{y}$ \\
$\mathrm{zy}$ & & & & \\
\hline
\end{tabular}

(31) and anonymity contradict (29); we were led to this by assuming there exists a rule satisfying (1), so no such rule exists.

Pareto implies $\mathrm{x} \succ \mathrm{y}$ whenever both individuals prefer $\mathrm{x}$ to $\mathrm{y}$. If the social welfare function $\mathrm{f}$ yields $\mathrm{y} \succ \mathrm{x}$ at every other profile then $\psi(\{x, y\})=\{x, y\}$. But we know that $\psi(\{x, y\})=\{x, y, z\}$ and thus $x \geq y$ must hold at some profile at which one person prefers $y$ to $x$. We have just shown that this is impossible if $\psi$ is relevance map \#1 and the associated social welfare function is transitive-valued and satisfies Pareto and anonymity on a full domain. We conclude by showing that this is also true in the case of relevance map \#6. 


\section{Relevance Map \#6}

As we noted above, relevance map \#6

\begin{tabular}{|c|c|}
\hline $\mathrm{S}$ & $\psi(\mathrm{S})$ \\
\hline$\{\mathrm{x}, \mathrm{y}\}$ & $\{\mathrm{x}, \mathrm{y}, \mathrm{z}\}$ \\
$\{\mathrm{y}, \mathrm{z}\}$ & $\{\mathrm{y}, \mathrm{z}, \mathrm{w}\}$ \\
$\{\mathrm{x}, \mathrm{w}\}$ & $\{\mathrm{x}, \mathrm{w}, \mathrm{y}\}$ \\
$\{\mathrm{y}, \mathrm{w}\}$ & $\{\mathrm{y}, \mathrm{w}, \mathrm{z}\}$ \\
$\{\mathrm{z}, \mathrm{w}\}$ & $\{\mathrm{z}, \mathrm{w}, \mathrm{y}\}$ \\
$\{\mathrm{x}, \mathrm{z}\}$ & $\{\mathrm{x}, \mathrm{z}, \mathrm{w}\}$ \\
& \\
\hline
\end{tabular}

is not isomorphic with the first and requires separate treatment. Reconsider the table

\begin{tabular}{|c|c|c|c|c|c|c|c|c|}
\hline $\mathrm{u}_{1}$ & $\mathrm{u}_{2}$ & $\mathrm{u}_{3}$ & $\mathrm{u}_{4}$ & $\mathrm{u}_{5}$ & $\mathrm{u}_{6}$ & $\mathrm{u}_{7}$ & $\mathrm{u}_{8}$ & $\mathrm{u}_{9}$ \\
\hline $\mathrm{z} \mathrm{z}$ & $\mathrm{z} \mathrm{y}$ & $\mathrm{z} \mathrm{y}$ & $\mathrm{x} \mathrm{z}$ & $\mathrm{x} \mathrm{y}$ & $\mathrm{x} \mathrm{y}$ & $\mathrm{x} \mathrm{z}$ & $\mathrm{x} \mathrm{y}$ & $\mathrm{x} \quad \mathrm{y}$ \\
$\mathrm{x} \mathrm{y}$ & $\mathrm{x} \mathrm{z}$ & $\mathrm{x} \mathrm{x}$ & $\mathrm{z} \mathrm{y}$ & $\mathrm{z} \mathrm{z}$ & $\mathrm{z} \mathrm{x}$ & $\mathrm{y} \mathrm{y}$ & $\mathrm{y} \mathrm{z}$ & $\mathrm{y} \mathrm{x}$ \\
$\mathrm{y} \mathrm{x}$ & $\mathrm{y} \mathrm{x}$ & $\mathrm{y} \mathrm{z}$ & $\mathrm{y} \mathrm{x}$ & $\mathrm{y} \mathrm{x}$ & $\mathrm{y} \mathrm{z}$ & $\mathrm{z} \mathrm{x}$ & $\mathrm{z} \mathrm{x}$ & $\mathrm{z} \mathrm{z}$ \\
\hline
\end{tabular}

Again, for at least one of these subprofiles, we may assume $\mathrm{x} \succeq \mathrm{y}$ and our first step is to show that we need only consider the sixth profile on the list.

Proposition 4: For each subprofile $\mathrm{u}_{\mathrm{i}}$ in the table, if $\mathrm{x} \succeq \mathrm{y}$ in the social ranking at $\mathrm{u}_{\mathrm{i}}$, then we also have $\mathrm{x} \succeq \mathrm{y}$ in the social ranking at $\mathrm{u}_{6}$.

Proof: This time start with the subprofile $\mathrm{u}_{2}$ and assume $\mathrm{x} \succeq \mathrm{y}$ in the social ranking there:

(1) $\mathrm{zy}$

$\mathrm{xz} \rightarrow \mathrm{x} \succeq \mathrm{y}$.

yx 
then derive:

(2)

\begin{tabular}{|l|l|l|l|l|}
\hline zy & & & & \\
$\mathrm{xw}$ & $\mathrm{x} \geq \mathrm{y}$ & $\mathrm{y} \succ \mathrm{w}$ & $\mathrm{xy}$ \\
$\mathrm{yz}$ & by (1) & by (P) & $\mathrm{x} \succ \mathrm{w}$ & $\mathrm{yw} \rightarrow \mathrm{x} \succ \mathrm{w}$ \\
$\mathrm{wx}$ & & & $\mathrm{wx}$ \\
\hline
\end{tabular}

(3)

\begin{tabular}{|l|l|l|l|l|}
\hline zy & & & & \\
$\mathrm{xw}$ & $\mathrm{x}>\mathrm{w}$ & $\mathrm{z} \succ \mathrm{x}$ & $\mathrm{zy}$ & $\mathrm{yw} \rightarrow \mathrm{z} \succ \mathrm{w}$ \\
$\mathrm{yz}$ & by (2) & by (P) & $\mathrm{z} \succ \mathrm{w}$ & $\mathrm{wz}$ \\
$\mathrm{wx}$ & & & & \\
\hline
\end{tabular}

(4)

\begin{tabular}{|l|l|l|l|l|}
\hline zy & & & & \\
yw & z $>$ w & w $\succ \mathrm{x}$ & & $\mathrm{zw}$ \\
$\mathrm{wx}$ & by (3) & by (P) & $\mathrm{z} \succ \mathrm{x}$ & $\mathrm{wx} \rightarrow \mathrm{z} \succ \mathrm{x}$ \\
$\mathrm{xz}$ & & & $\mathrm{xz}$ \\
\hline
\end{tabular}

(5)

\begin{tabular}{|l|l|l|l|l|}
\hline zw & & & & \\
wx & $\mathrm{z} \succ \mathrm{x}$ & $\mathrm{x} \succ \mathrm{y}$ & $\mathrm{zw}$ \\
$\mathrm{xy}$ & by (4) & by (P) & $\mathrm{z} \succ \mathrm{y}$ & $\mathrm{wy} \rightarrow \mathrm{z} \succ \mathrm{y}$ \\
$\mathrm{yz}$ & & & $\mathrm{yz}$ \\
\hline
\end{tabular}


(6)

\begin{tabular}{|l|c|c|l|l|}
\hline xw & & & & \\
zy & $\mathrm{z} \succ \mathrm{y}$ & $\mathrm{x} \succ \mathrm{z}$ & $\mathrm{xy}$ \\
$\mathrm{wx}$ & by (5) & by (P) & $\mathrm{x} \succ \mathrm{y}$ & $\mathrm{zx} \rightarrow \mathrm{x} \succ \mathrm{y}$ \\
$\mathrm{yz}$ & & & $\mathrm{yz}$ \\
\hline
\end{tabular}

(6) tells us $\mathrm{x} \succ \mathrm{y}$ at $\mathrm{u}_{6}$.

Now consider any subprofile in the table, other than $\mathrm{u}_{2}$ or $\mathrm{u}_{6}$. Starting with that subprofile, assume $\mathrm{x} \geq \mathrm{y}$. Then insert $\mathrm{w}$ just below $\mathrm{y}$ for each individual, to get both $\mathrm{x} \geq \mathrm{y}$ and, by Pareto, $\mathrm{y} \succ \mathrm{w}$. By transitivity, $\mathrm{x} \succ \mathrm{w}$. Restricted to $\{\mathrm{x}, \mathrm{y}, \mathrm{w}\}$, this profile has

\section{xy}

yw

wx

Thus we have established step (2) above, and steps (3) to (6) then imply $\mathrm{x}>\mathrm{y}$ at $\mathrm{u}_{6}$.

Proposition 5: There is no rule satisfying all of ISA, Pareto, full domain, transitivity, and anonymity with relevance map \#6 such that

$$
\begin{aligned}
& x y \\
& z x \rightarrow x \geq y
\end{aligned}
$$

yz

Proof:

We are going to assume such a rule does exist and derive an inconsistency. We start of course from

(1)

$$
\begin{aligned}
& x y \\
& z x \rightarrow x \geq y \\
& y z
\end{aligned}
$$


(2)

\begin{tabular}{|l|c|c|l|l|}
\hline xy & & & & \\
zw & $\mathrm{x} \geq \mathrm{y}$ & $\mathrm{y} \succ \mathrm{w}$ & $\mathrm{xy}$ \\
$\mathrm{yx}$ & by (1) & by (P) & $\mathrm{x} \succ \mathrm{w}$ & $\mathrm{yw} \rightarrow \mathrm{x} \succ \mathrm{w}$ \\
$\mathrm{wz}$ & & & \\
\hline
\end{tabular}

(3)

\begin{tabular}{|l|c|c|l|l|}
\hline wy & & & & \\
$\mathrm{xw}$ & $\mathrm{x} \geq \mathrm{y}$ & $\mathrm{w} \succ \mathrm{x}$ & & $\mathrm{wy}$ \\
$\mathrm{zx}$ & by (1) & by (P) & $\mathrm{w} \succ \mathrm{y}$ & $\mathrm{zw} \rightarrow \mathrm{w} \succ \mathrm{y}$ \\
$\mathrm{yz}$ & & & $\mathrm{yz}$ \\
\hline
\end{tabular}

(4)

\begin{tabular}{|l|l|l|l|l|}
\hline zy & & & & \\
$\mathrm{xw}$ & $\mathrm{x}>\mathrm{w}$ & $\mathrm{z} \succ \mathrm{x}$ & $\mathrm{zy}$ \\
$\mathrm{yz}$ & by (2) & by (P) & $\mathrm{z} \succ w$ & $\mathrm{yw} \rightarrow \mathrm{z} \succ \mathrm{w}$ \\
$\mathrm{wx}$ & & & $\mathrm{wz}$ \\
\hline
\end{tabular}

(5)

\begin{tabular}{|l|c|c|l|l|}
\hline wy & & & & \\
zx & w $>\mathrm{y}$ & $\mathrm{y}>\mathrm{x}$ & & $\mathrm{wy}$ \\
$\mathrm{yw}$ & by (3) & by (P) & $\mathrm{w}>\mathrm{x}$ & $\mathrm{yx} \rightarrow \mathrm{w} \succ \mathrm{x}$ \\
$\mathrm{xz}$ & & & & \\
\hline
\end{tabular}


(6)

\begin{tabular}{|l|l|l|l|l|}
\hline zy & & & & \\
$\mathrm{yw}$ & $\mathrm{z} \succ \mathrm{w}$ & $\mathrm{w} \succ \mathrm{x}$ & $\mathrm{zw}$ \\
$\mathrm{wx}$ & by (4) & by (P) & $\mathrm{z} \succ \mathrm{x}$ & $\mathrm{wx} \rightarrow \mathrm{z} \succ \mathrm{x}$ \\
$\mathrm{xz}$ & & & $\mathrm{xz}$ \\
\hline
\end{tabular}

(7)

\begin{tabular}{|l|l|l|l|l|}
\hline wy & & & & \\
yx & w $>\mathrm{x}$ & $\mathrm{x}>\mathrm{z}$ & & wy \\
$\mathrm{xz}$ & by (5) & by (P) & $\mathrm{wz}>\mathrm{z}$ & $\mathrm{yz}>\mathrm{w}$ \\
$\mathrm{zw}$ & & & & \\
\hline
\end{tabular}

(8)

\begin{tabular}{|l|c|c|l|l|}
\hline zw & & & & \\
wx & $\mathrm{z} \succ \mathrm{x}$ & $\mathrm{x} \succ \mathrm{y}$ & & $\mathrm{zw}$ \\
$\mathrm{xy}$ & by (6) & by (P) & $\mathrm{z} \succ \mathrm{y}$ & $\mathrm{wy} \rightarrow \mathrm{z} \succ \mathrm{y}$ \\
$\mathrm{yz}$ & & & $\mathrm{yz}$ \\
\hline
\end{tabular}

(9)

\begin{tabular}{|l|c|c|l|l|}
\hline xy & & & & \\
wz & w $>\mathrm{z}$ & $\mathrm{x} \succ \mathrm{w}$ & $\mathrm{xz}$ \\
$\mathrm{yx}$ & by (7) & by (P) & $\mathrm{x} \succ \mathrm{z}$ & $\mathrm{wx} \rightarrow \mathrm{x} \succ \mathrm{z}$ \\
$\mathrm{zw}$ & & & $\mathrm{zw}$ \\
\hline
\end{tabular}


(10)

\begin{tabular}{|l|c|c|l|l|}
\hline yz & & & & \\
$\mathrm{xy}$ & $\mathrm{x} \succ \mathrm{z}$ & $\mathrm{y} \succ \mathrm{x}$ & & $\mathrm{yz}$ \\
$\mathrm{wx}$ & by (9) & by (P) & $\mathrm{y} \succ \mathrm{z}$ & $\mathrm{wy} \rightarrow \mathrm{y} \succ \mathrm{z}$ \\
$\mathrm{zw}$ & & & $\mathrm{zw}$ \\
\hline
\end{tabular}

As an intermediate result, we want to establish

$$
\begin{aligned}
& x y \\
& z z \rightarrow y \succ x . \\
& y x
\end{aligned}
$$

We will prove this by assuming

(11)

$$
\begin{aligned}
& \mathrm{xy} \\
& \mathrm{zz} \rightarrow \mathrm{x} \geq \mathrm{y} \\
& \mathrm{yx}
\end{aligned}
$$

and showing that that leads to a contradiction.

(12)

\begin{tabular}{|l|c|c|l|l|}
\hline $\mathrm{xy}$ & & & & \\
$\mathrm{zw}$ & $\mathrm{x} \geq \mathrm{y}$ & $\mathrm{y}>\mathrm{z}$ & $\mathrm{xw}$ \\
$\mathrm{yz}$ & by (11) & by $\left(10^{*}\right)$ & $\mathrm{x} \succ \mathrm{z}$ & $\mathrm{zz} \rightarrow \mathrm{x}>\mathrm{z}$ \\
$\mathrm{wx}$ & & & $\mathrm{wx}$ \\
\hline
\end{tabular}


(13)

\begin{tabular}{|l|c|c|l|l|}
\hline yw & & & & \\
xz & $\mathrm{x}>\mathrm{z}$ & $\mathrm{y} \succ \mathrm{x}$ & & $\mathrm{yw}$ \\
$\mathrm{zy}$ & by (12) & by (P) & $\mathrm{y} \succ \mathrm{z}$ & $\mathrm{zz} \rightarrow \mathrm{y} \succ \mathrm{z}$ \\
wx & & & wy \\
\hline
\end{tabular}

(14)

\begin{tabular}{|l|c|c|l|l|}
\hline wy & & & & \\
$\mathrm{xz}$ & $\mathrm{x} \geq \mathrm{y}$ & $\mathrm{z} \succ \mathrm{x}$ & $\mathrm{wy}$ \\
$\mathrm{zw}$ & by (11) & by $\left(6^{*}\right)$ & $\mathrm{z} \succ \mathrm{y}$ & $\mathrm{zz} \rightarrow \mathrm{z} \succ \mathrm{y}$ \\
$\mathrm{yx}$ & & & $\mathrm{yw}$ \\
\hline
\end{tabular}

(14) and anonymity contradict (13); we were led to this by assuming (11) which must be false, so we have

$$
\begin{aligned}
& x y \\
& z z \rightarrow y \succ x .
\end{aligned}
$$

yx

Now return to our main line of derivations where we will quickly find a final inconsistency.

\begin{tabular}{|l|c|c|l|l|}
\hline xy & & & & \\
wz & $y>x$ & $x>z$ & wy \\
zx & by (15) & by (9) & $\mathrm{y} \succ \mathrm{z}$ & $\mathrm{zz} \rightarrow \mathrm{y}>\mathrm{z}$ \\
$\mathrm{yw}$ & & & $\mathrm{yw}$ \\
\hline
\end{tabular}


(17)

\begin{tabular}{|l|c|c|l|l|}
\hline wx & & & & \\
zy & y $>\mathrm{z}$ & $\mathrm{x} \succ \mathrm{y}$ & $\mathrm{wx}$ \\
$\mathrm{xz}$ & by (16) & by (P) & $\mathrm{x} \succ \mathrm{z}$ & $\mathrm{zz} \rightarrow \mathrm{x} \succ \mathrm{z}$ \\
$\mathrm{yw}$ & & & $\mathrm{xw}$ \\
\hline
\end{tabular}

(18)

\begin{tabular}{|l|c|c|l|l|}
\hline wx & & & \\
yz & $y>x$ & $z \succ y$ & & wx \\
zw & by $\left(15^{*}\right)$ & by $\left(8^{*}\right)$ & $\mathrm{z} \succ \mathrm{x}$ & $\mathrm{zz} \rightarrow \mathrm{z} \succ \mathrm{x}$ \\
$\mathrm{xy}$ & & & $\mathrm{xw}$ \\
\hline
\end{tabular}

Because (18) contradicts (17) we must drop the assumption that there exists a rule satisfying (1); no such rule exists.

\section{Larger numbers}

It remains to show how far our analysis can be generalized to larger numbers of individuals and alternatives. It is straightforward to see that a proof for $n=2$ implies a corresponding theorem for any even number of individuals. Suppose $\mathrm{n}=2 \mathrm{r}$ and that there exists a rule $\mathrm{F}$ for $\mathrm{n}$ satisfying all of: ISA, Pareto, full domain, transitivity, and anonymity. Then for just two individuals, define a rule $\mathrm{f}$ that at profile $\mathrm{u}$ yields $\mathrm{F}(\mathrm{u}, \mathrm{u}, \ldots, \mathrm{u})$ at $\mathrm{r}$ copies of $\mathrm{u}$. Rule $\mathrm{f}$ will inherit ISA, Pareto, full domain, transitivity, and anonymity from $F$. Since we have shown no such rule $f$ exists, there must not be any for $n$ $=2 \mathrm{r}$. 


\section{References}

Campbell, D. E. and J. S. Kelly (2000) 'Information and Preference Aggregation,' Social Choice and Welfare 17: 3-24.

Campbell, D. E. and J. S. Kelly (2002) 'Impossibility Theorems in the Arrovian Framework' Handbook of Social Choice and Welfare, edited by K. J. Arrow, A. K. Sen, and K. Suzumura (Amsterdam: North-Holland) 35-94.

Campbell, D. E. and J. S. Kelly (2004) 'Social Welfare Functions that Satisfy Pareto, Anonymity, and Neutrality, but not IIA,'Social Choice and Welfare, this issue. 